\title{
Assessment of the model refinement category in CASP12
}

Ladislav Hovan, ${ }^{1, *}$ Vladimiras Oleinikovas, ${ }^{1, *}$ Havva Yalinca, ${ }^{1, *}$ Andriy

Kryshtafovych, ${ }^{2}$ Giorgio Saladino, ${ }^{1}$ and Francesco Luigi Gervasio ${ }^{1,3}$

${ }^{1}$ Department of Chemistry, University College London, London WC1E 6BT, United

Kingdom

${ }^{2}$ Genome Center, University of California, Davis, California 95616, USA

${ }^{3}$ Institute of Structural and Molecular Biology, University College London, London WC1E 6BT, United Kingdom.

* Contributed equally.

Correspondence:

Francesco Luigi Gervasio, Department of Chemistry, University College London, London WC1E 6BT, United Kingdom.

Email:f.l.gervasio@ucl.ac.uk 


\begin{abstract}
We here report on the assessment of the model refinement predictions submitted to the 12th Experiment on the Critical Assessment of Protein Structure Prediction (CASP12). This is the fifth refinement experiment since CASP8 (2008) and, as with the previous experiments, the predictors were invited to refine selected server models received in the regular (nonrefinement) stage of the CASP experiment. We assessed the submitted models using a combination of standard CASP measures. The coefficients for the linear combination of $Z$-scores (the CASP12 score) have been obtained by a machine learning algorithm trained on the results of visual inspection. We identified 8 groups that improve both the backbone conformation and the side chain positioning for the majority of targets. Albeit the top methods adopted distinctively different approaches, their overall performance was almost indistinguishable, with each of them excelling in different scores or target subsets. What is more, there were a few novel approaches that, while doing worse than average in most cases, provided the best refinements for a few targets, showing significant latitude for further innovation in the field.
\end{abstract}




\section{INTRODUCTION}

The refinement category of CASP (Critical Assessment of protein Structure Prediction) was introduced in the eighth round of the experiment to address the challenge of refining selected server models from the regular CASP experiment to better represent the native structure. ${ }^{1}$

It is now recognized that a number of applications, ranging from rational drug discovery to crystallography (molecular replacement) require high accuracy models..$^{2-7}$ Thus, a reliable computational approach for refinement is highly desirable. In this respect, the CASP refinement experiment can be very useful, by highlighting the best practices and progress in the field.

As discussed in previous CASP experiments ${ }^{7}$, refinement is challenging, as predictors need to further improve the best server models both on a global and local scale. Indeed, since many of the starting models are already close to the native structures, the refinement methods need to be exquisitely sensitive to introduce subtle changes into the original structures, such as repacking of side chains. At the same time, these methods should be also capable of significant rearrangement of proteins, including rerouting of the backbone or changes to the secondary structure elements.

Many methods (i.e. CASP groups ${ }^{1}$ ) participating in the mainstream CASP prediction already perform some degree of refinement, although the time constraint might prevent the use of computationally-intensive strategies. Analogously, a few groups participating in the refinement challenge might attempt a partial reconstruction of the fold from the sequence, blurring the distinction between the traditional modeling and refinement. Finding the balance between small changes that only locally perturb the starting structure, and more substantial rearrangements that refold whole sub-domains is the essence of the refinement task itself. As

${ }^{1}$ Following the usual CASP convention, we refer to methods as groups (or CASP groups). When we refer to authors, we use the term "research group". 
refinement assessors have already pointed out in the past, ${ }^{7}$ improving more fine-grained features of model quality such as side chain positioning and physicality is seldom useful without a correct backbone positioning. This turned out to be the case also in CASP12.

Historically, a broad variety of methods have been used in protein structure refinement, ranging from knowledge-based and fragment-based approaches to molecular dynamics (MD) with physics-based force fields. ${ }^{8-15}$ Lately, due to increasingly accurate physics-based force fields ${ }^{10,16-}$ ${ }^{21}$ and faster parallel computers and GPUs, MD in combination with physics-based force fields and smart constraints is increasingly used in successful refinement pipelines. ${ }^{11}$ Starting from a clear disadvantage, where such approaches would in most cases make the starting model worse, they have been so successful that they are now used in most top-performing CASP12 methods. Eight out of top ten CASP12 refinement methods use MD with recently developed physics-based potentials (alone or in combination with other approaches), while the remaining two use MD with a hybrid knowledge-based / physics-based potential. The increasing success of MD methods might seem unsurprising given that they have been shown to reversibly fold small proteins, recovering their native structure. ${ }^{22}$ However, due to a combination of computationally prohibitive sampling times (especially in the context of CASP), missing information on structuredetermining contacts (e.g. cofactors, ligands and protein-protein contacts) as well as residual force-field inaccuracies, refinement with MD simulations works best when light positional restraints are applied to the starting structure..$^{10}$ The strength of these restraints appear to be a crucial parameter for a successful refinement and concurs to differentiate the performance of the best approaches in the CASP refinement challenge. The use of restraints might also explain the difficulties encountered by MD-only pipelines in refining starting models that are far (in terms of GDT_HA and RMSD) from the target. 
Enhanced sampling algorithms, which are increasingly used to address the time-scale problem of atomistic MD simulations ${ }^{23-26}$, also allow the inclusion of heuristic information about protein folds without the need for explicit constraints. Approaches such as MELD (Modeling Employing Limited Data) ${ }^{26}$, which sample important conformational states by combining structural and heuristic information with atomistic MD simulations, show promise.

As an alternative to physics-based force-fields, knowledge-based potentials are highly attractive for structure refinement, due to their more direct relation with protein structures and lower computational cost. ${ }^{27-29}$ They are generally derived from knowledge of atom- or residue-specific interactions and have been shown to be able to refine near-native structures. ${ }^{30}$

As the CASP12 refinement targets span a wide range of sizes (from 54 to 396 residues) and accuracies of starting models (GDT_HA scores ranging from 23 to 76), a battery of numerical evaluation measures sensitive both to subtle local changes and more global structural features needed to be used. For a well-rounded assessment, we combined conceptually different evaluation measures tested in previous CASP editions. The coefficients used to combine various accuracy metrics have been automatically determined with genetic algorithms and a Monte Carlo approach to best correlate with the ranking obtained by visual inspection. Reassuringly, the CASP12 score is robust upon restraining individual metrics to less than $20 \%$. The ranking of the top 10 methods is very similar to that obtained by applying the CASP11 and CASP10 scores (Table S1). ${ }^{7,32}$ Still, we believe that the visual inspection of a considerable fraction of submissions enabled us to better distinguish subtle differences in the submitted structures and reward the most successful ones. Overall, the analysis of the CASP12 refinement predictions shows a consolidation of the trend started in CASP10 and continued in CASP11. The top methods excel in a number of metrics, but the prediction accuracy is in many 
respects similar across different methods, and for most targets incremental. While some methods are relatively more conservative, providing a reliable but small refinement, other approaches are more adventurous providing significant improvement of the global and local structure for some targets while making a few others worse. Finally, a few new approaches that trail the top scoring methods for most targets, occasionally provided striking refinements.

\section{MATERIALS AND METHODS}

\section{Target selection}

The overall setup of the refinement experiment in CASP12 is similar to previous CASPs $^{1,7,31,32}$. Refinement targets are selected from amongst the main challenge targets. One of the major concerns of the organizers and assessors is to provide as many interesting targets as possible, while discarding cases where the experimental structure is dictated by extensive multimeric interactions, or where submitted models are already good and not much room is left for the refinement, or, on the contrary, where the models are too poor and the improvement would require substantial conformational rearrangements in the global structure. After careful analysis and visual inspection, we selected a total of 42 targets for the CASP12 refinement challenge. Of these, $23(55 \%)$ were easier tertiary structure prediction targets (or TBM, see the domain classification paper elsewhere in this issue), 13 (31\%) were intermediate (or TBM/FM) and $6(14 \%)$ were difficult for tertiary structure prediction targets (FM). Differently from CASP11, a large portion of CASP12 refinement targets were the FM and TBM/FM targets, which were included in the scope of the refinement experiment to follow the recent progress in the free modeling prediction and test the abilities of the 
refinement methods on this kind of targets. Also, some targets in CASP12 were considerably larger than those in previous CASP refinement experiments, likely contributing to the difficulty of their refinement. As in previous CASP experiments, for each target, one of the best server-submitted models was suggested as the starting model for the refinement. The accuracy of the model's backbone (GDT_HA score, the main criterion) and its compliance to the known protein stereochemical rules (MolProbity score, secondary criterion) were used in the selection process. The accuracy scores for the initial structures are reported in Table 1, together with their CASP IDs.

Many targets for the CASP12 main challenge are large multi-domain complexes. This makes the refinement of the corresponding single domain structures very challenging, as many inter-domain contacts might stabilize the observed X-ray arrangement. In order to retain as many targets as possible while providing a rigorous assessment, we removed from the evaluation some regions of targets clearly affected by inter-domain and protein-protein contacts (pertaining to TR866, TR868, TR870, TR876, TR885, TR887 and TR922). In TR866, for example, the C-terminal helix (V142-Y152) at the oligomerization interface is stabilized by contacts with the other proteins forming a hexamer and therefore was excluded from the evaluations. Analogously, in TR868, which is a dimer of heterodimers, residues Q65-F75 were removed since they form part of the dimerization interface. A visual summary of the regions removed from the seven targets is reported in Figure 1. Target TR887 is a special case of a swapped dimer in the X-ray structure. Refining the swap segment in the absence of the other monomer (Figure 1) and of correctly swapped templates, makes little sense. Instead, when defining the target structure of TR887, we replaced the region with the respective swapped segment from the second monomer (in green in Figure 1), as predicted by the servers in the main category. 


\section{Model accuracy measures}

A number of accuracy scores are automatically calculated by the Protein Structure Prediction Center; we refer to the documentation therein for further details. ${ }^{33,34}$ Here, in line with previous CASP refinement experiments, we based our analysis on the following metrics: the Root Mean Square Deviation of the $\mathrm{C}_{\alpha}$ atoms (RMSD), the Global Distance Test ${ }^{35}$ (GDT_TS and GDT_HA), SphereGrinder ${ }^{36}$ (SphGr), the Local Distance Difference Test (LDDT) ${ }^{37}$ and the MolProbity score (MolPrb) ${ }^{38}$ to assess the model stereochemistry. In addition to these, we also considered the Contact Area Difference score (CAD) ${ }^{39}$ and the Quality Control Score (QCS) ${ }^{40}$ The $\mathrm{C}_{\alpha}$ RMSD measure calculates the average distance between the corresponding $\mathrm{C}_{\alpha}$ atoms in the model and target after their optimal least-square superposition. The GDT_TS score is a measure of model's backbone accuracy. The model is superposed (using LGA ${ }^{33}$ ) to the target structure in multiple trials, maximizing the number of $\mathrm{C}_{\alpha}$ atoms within a certain cut-off distance. The GDT_TS value is the average percentage of well-fit $\mathrm{C}_{\alpha}$ atoms calculated at four different cut-off values $(1.0,2.0,4.0$ and $8.0 \AA)$. The GDT_HA is the high-accuracy version of the latter metric, for which the cut-offs are halved. SphereGrinder is a local-based measure of similarity introduced in CASP10. For every residue, the RMSD score is calculated on sets of corresponding atoms inside the spheres of a selected radius ( $6 \AA$ in CASP) centered on the same $\mathrm{C} a$ atoms in the model and target. The average percentage of spheres fitting under $2 \AA$ and $4 \AA$ RMSD cutoffs is reported as the SG-score. The LDDT is a superposition-free measure based on the comparison of all-atom distance maps of model and target structures. For each residue pair within a distance cutoff of $15 \AA$, the difference between the corresponding distances in the model and in the target is calculated. The LDDT score reports the average fraction of the differences below four different threshold values $(0.5,1,2$, and $4 \AA)$. The CAD- 
score $^{39}$ is based on a similar concept, but uses the difference in residue-residue contact surfaces as derived by the Voronoi tessellation, instead of a set of cut-offs. The QCS score was developed by Grishin and co-workers ${ }^{40}$ as a result of their experience as evaluators of the CASP9 free modeling (FM) category and is the average of six individual scores that take into account the length, position and reciprocal orientation of secondary structure elements and $\mathrm{C}_{\alpha}-\mathrm{C}_{\alpha}$ contacts. We observed that adding QCS to the final score led to a better agreement with the manually curated rankings. Finally, the MolProbity score is a target-independent measure of the model stereochemistry accuracy. It is derived from an extensive analysis of deposited PDB structures ${ }^{38}$ and reports the presence of clashes, the rotameric state of the side-chains, and the number of residues with backbone torsions $\varphi$ and $\psi$ outside the highdensity Ramachandran regions.

The scatter plot and correlation of the different metrics for all the submissions is reported in Figure 2. Since most qūality scores are highly correlated, after extensive trials with our machine learning algorithm trained on the ranking obtained from our manually curated evaluations, we retained the RMSD, GDT_HA, SphGr, QCS and MolPrb in our final score (see below).

For ranking purposes, all the metrics were converted to Z-scores in a two-step procedure. First, Z-score were calculated from the distribution of raw scores for all models submitted on a target. Then, models with a $Z$-score lower than -2 were excluded and $Z$-scores were recalculated based on the mean and standard deviation of the outlier-free model set. Finally, models with Z-scores lower than -2 (in both calculation stages) were assigned a value of -2 in order not to over-penalize the groups attempting novel strategies. The cumulative ranking scores were obtained by summing the target-based Z-scores for each group, assigning a Zscore of -2 every time a target was not predicted by a particular group. 
To estimate the overall added value of the refinement, we compared the results of CASP12 groups to those of the artificial 'naïve group', which always resubmits unchanged starting model, as proposed by the past CASP assessors. ${ }^{7}$

\section{ASSESSMENT OF THE CASP12 REFINEMENT CATEGORY RESULTS}

\section{General Overview of targets and predictions}

A total of 39 groups took part in the CASP12 refinement challenge. 20 groups submitted predictions for all the 42 targets and further seven for 41 targets. CASP12 refinement results spanned a wide range of scores. Figure 3 shows general statistics of the accuracy of first submitted models with respect to the accuracy of the corresponding starting models (in terms of GDT_HA) for targets of different lengths and different accuracies of starting models. The best potential for improvement was observed for smaller targets (top left panel) and those in the medium range of starting GDT_HAs (bottom middle panel). Overall, $34 \%$ of the models improved over the initial structure. A similar conclusion can be drawn from the $\triangle$ RMSD-based analysis (see Figure S1). As expected, the median score change is close to zero, with $\sim 70 \%$ of the predictions having a $\Delta$ GDT_HA between -5 and 5 (Figure 3). This suggests a prevalence of conservative predictions. While more potential for worsening the starting structure is observed, with $23 \%$ of models taking a loss of 5 or more GDT_HA units, $3 \%$ of the models showed remarkable examples of refinement improving starting structures by 5 to 20 GDT_HA units.

\section{Performance of methods according to various}




\section{assessment measures}

We used several conceptually different measures for the refinement assessment (see Methods). This allowed us to analyze models from different perspectives (e.g., overall accuracy of the backbone, all-atom accuracy of local substructures or stereochemical accuracy).

Figure 4 shows the results according to one of the most widely used CASP measures, GDT_HA, which is used here to evaluate accuracy of the protein backbone refinement. Using this metric, it is clear that a number of groups are able on average to refine the targets, with the best groups significantly refining some targets, while still worsening a few. Eight groups SVMQA, Seok, FEIG, GOAL_COMPLEX, BAKER, Kiharalab, Seok-server and GOAL - on average improve over the starting model (top panel). The largest average accuracy increase, however, is very modest and constitutes only 1.3 GDT_HA points (by the SVMQA group). In the bottom three panels we report the results binned for different accuracies of starting models. In contrast to the general results (showing that the highest improvement potential is for targets with medium-range starting GDT_HAs), the top performing groups achieved better results on targets with low initial GDT_HA scores. For example, BAKER, the best group on targets with starting GDT_HA<40, improved the GDT_HA by 2 units on average. The average refinement of structures close to the target (high initial GDT_HA) is more modest, even though 10 out of 39 groups stayed in the positive average $\Delta$ GDT_HA territory.

We also performed the analyses similar to the one reported in Figure 4 according to all other evaluation measures. In Figure S2 we show the distributions of GDT_TS scores of refined models (model 1) for all CASP12 refinement targets. Figures S3 and S4 show examples of the analyses according to the RMSD_CA and GDT_TS metrics. It can be seen that the results are conceptually similar to the GDT_HA-based data - a number of groups are able to improve on 
average, but in most cases only marginally.

To analyze the relative performance of CASP12 groups, we converted raw evaluation scores into the corresponding Z-scores (see Methods). Figures 5 and S5 illustrate the performance of the participating groups as measured by the normalized RMSD, GDT_HA, SphGr, QCS and MolPrb scores (Figure 5) and normalized GDT_TS, LDDT and CAD scores (Figure S5). While the ranking changes when different metrics are used, some CASP groups remain consistently in the top. These include the following four groups: GOAL (from J. Lee's research group), Seok (from C. Seok's research group), BAKER (from D. Baker's research group) and FEIG (from M. Feig's research group).

Figure 6 reports how many times the best CASP 12 groups appear among the best 10 according to eight evaluation metrics (RMSD, GDT_HA, GDT_TS, SphGr, LDDT, CAD, QCS and MolPrb). Seok and GOAL have the best cumulative score according to three metrics each: GDT_TS, LDDT and CAD for Seok; RMSD, QCS and SphGr for GOAL. FEIG and BAKER appear as the best in one metric each (GDT_HA and MolPrb, respectively). Some methods such as SVMQA, LEE, LEEab GOAL_COMPLEX (all from J. Lee's research group and similar to GOAL) and Seok-server (similar to Seok) as well as FLOUDAS REFINESERVER (herein referred to as FLOUDAS_REFI), Kiharalab and STAP also appear among the first 10 groups. Moreover, BAKER, Seok, Seok-server and SVMQA on average perform better than the "naïve method", regardless of the metric used. The "best 10 " methods according to each single metric are reported in SI Figure S6.

\section{The CASP12 Score}

The assessor's formula for ranking groups in CASP is generally defined as a combination of 
different metrics. As discussed in previous CASP experiments, ${ }^{7,32}$ this is due to two considerations: single standard scores, such as RMSD and GDT_HA, may miss promising models ${ }^{40,41}$ and different metrics may suggest different rankings (as shown in Figures S6-S8). Examples of discrepancies in rankings according to different metrics are shown in Figure 7 for targets TR882 and TR948. It can be seen that different models scored very high with respect to either SphGr (left) or GDT_HA (right) metrics, and at the same time very low with respect to the other score.

In CASP12 we had a high percentage of FM and FM/TBM targets (45\%). Manual inspection $^{41}$ has been traditionally used to evaluate this kind of targets in CASP. The main reason behind this was the reduced reliability of standard scores in FM. It is for instance well known that GDT_HA score may give preference to models containing small but precisely modeled substructures over those with a good topology and general fold. ${ }^{40,41}$ Manual assessment, on the contrary, is better suited to assess the general fold, the relative arrangement of secondary structure elements and local hydrogen bond networks. However, it requires significant time investment and might suffer from being subjective.

In order to benefit from manual assessment while minimizing the pitfalls of subjectiveness and avoiding the definition of arbitrary weights for the different metrics, we used a machine learning approach to devise a linear combination of standard scores based on the visual inspection. Four assessors (LH, VO, HY and GS) visually inspected all "model 1" predictions for 14 targets $(33 \%)$ and each independently scored them. The visually inspected targets have the same fraction of FM, TBM/FM and TBM targets as the complete set of targets. The targets were divided in 2 to 4 regions (depending on the length of the target) and each evaluator carefully assessed their global and local structure, with specific focus on features 
(such as the length, position and reciprocal orientation of secondary structure elements and hydrogen bond networks) that might increase the usefulness of the refined structure for docking, molecular replacement, etc. The final scores from different evaluators (ranging from -5 to +5 ) were in remarkable agreement (see an example of target TR876 in Figure S9 in SI). We then converted the raw manual scores to standard $Z$-scores, and derived optimal weights $\hat{c}_{m}$ for $Z$-scores $Z_{m}$ of each metric $m$ in our subset, defining the final score $\hat{S}$ as:

$$
\hat{S}=\sum_{m=0}^{n} \hat{c}_{m} \cdot Z_{m}
$$

under the normalization condition: $\sum_{m=0}^{n} \hat{c}_{m}=1$, where $n$ is the number of metrics selected ( 8 in our case). For the purpose of optimizing the coefficients, we used both a Monte Carlo (MC) simulation and a genetic algorithm (GA). Ultimately, the GA was able to push the optimization further in preliminary analysis and was used exclusively. The procedure involves the iterative generation of $N=1000$ sets of trial coefficients $\tilde{c}_{i}=\left\{\tilde{c}_{m}\right\}_{i}$ (with $\left.i=1, \ldots, N\right)$ and defining a fitness function $f\left(\tilde{c}_{i}\right)$, which selects the best sets. In the first iteration, all 1000 sets are randomly generated. At every subsequent iteration, the best 5\% (50) sets of coefficients are retained and a further 250 random sets are randomly generated. To have an ensemble of 1000 sets again, $700\left\{c_{m}\right\}$ sets are generated by averaging the coefficients between randomly chosen pairs of sets. As we are interested in reproducing the ranking obtained with our manual assignment, we defined $f\left(\mathrm{c}_{i}\right)$ as the Spearman rank correlation coefficient calculated using the ranking resulting from our manual assignment and the one resulting using the current score:

$$
\tilde{S}_{i}=\sum_{m=0}^{n} \tilde{c}_{m, i} \cdot Z_{m}
$$


where the $\left\{\tilde{c}_{m}\right\}_{i}$ are the coefficients at the current step. After some trials with various combinations, we considered only the rank of the six best models according to our manual assignment and the proposed score, for every manually evaluated target. After 100 iterations the weights converged, resulting in formula:

$$
\hat{S}_{\mathrm{CASP} 12}=0.46 \cdot Z_{\mathrm{RMSD}}+0.17 \cdot Z_{\mathrm{GDT}_{-} \mathrm{HA}}+0.2 \cdot Z_{\mathrm{SphGr}}+0.15 \cdot Z_{Q C S}+0.02 \cdot Z_{M o l P r b}
$$

where the weights for $Z_{\mathrm{LDDT}}$ and $Z_{\mathrm{CAD}}$ were manually excluded from the final score as they were approaching zero and GDT_TS was very strongly correlated with GDT_HA.

As expected, the ranking obtained by the final $\hat{S}_{C A S P 12}$ score correlates better with the ranking obtained by visual inspection than those obtained by CASP10 and CASP11 scores (Figure S10). We were initially surprised by the high coefficient of the RMSD. However, the ranking is robust upon constraining RMSD to $20 \%$ or less. Fixing the coefficient for $Z_{\mathrm{RMSD}}$ at 0.2 and that for $Z_{\text {MolPrb }}$ at 0.02 , the ranking with this "restricted" CASP12 score is very similar to the original CASP12 ranking (see Table S2).

It should also be noted that the inclusion of MolProbity worsens the correlation with the manual assessment. By excluding $Z_{\text {MolPrb }}$ and re-optimizing the coefficients, the Spearman rank correlation coefficient increases to 0.491 from the 0.467 of $\hat{S}_{C A S P 12}$. This shows that the local stereochemistry of the models is not a priority for most groups. Thus, we decided to retain the MolProbity score, albeit with a small coefficient, to continue encouraging predictors to fix the local stereochemistry of their models.

The performance of different groups according to the $\hat{S}_{C A S P 12}$ score is reported in Figure 8 and Table 2. GOAL ranks narrowly first, followed by Seok and BAKER with almost indistinguishable scores, Seok_server (similar to Seok, see description below), 
SVMQA ( similar to GOAL) and FEIG. For comparison, the rankings on CASP10 and CASP11 assessor-defined scores are reported in Figure S11 and Table S1.

The final rankings by CASP12 and CASP11 scores are similar. For instance, only in 10 of the 42 targets does the highest scoring model 1 according to the CASP12 score differ from the highest scoring model according to the CASP11 score (Table S3). In 4 of these 10 cases the highest scoring models according to the CASP12 score are clearly more accurate than the highest scoring models according to the CASP11 score (Figures S12-S15), in one case the highest scoring model according to CASP11 is marginally better than the one of CASP12 and in the remaining six cases the difference is negligible. We also tested if applying the $\hat{S}_{\text {CASP12 }}$ score to CASP11 targets would yield different top models. For most targets, the top-ranking predictions are the same, with the exception of six targets (TR217, TR288, TR280, TR760, TR774, TR795). In three cases (TR217, TR288, TR280) the highest scoring model according to the CASP12 score are more accurate, while in the remaining three cases the differences are too small to be significant (see Table S4 for detailed analysis).

All top 10 refinement methods outperformed the naïve submission in CASP12 improving both the backbone conformation and the side chain positioning. The difference between the top 6 methods is very small. This is confirmed by both a pair-wise Wilcoxon signed-rank test and a global Friedman test that concur that their performances are marginally different.

Based on the results, we can roughly classify the methods in two categories: "conservative" and "adventurous". The conservative methods typically yield structures that are close to the initial ones and which score in a narrow range around the starting model's score. Seok and FEIG's approaches belong to this category. Adventurous methods seem to have the ability to substantially improve the structures in some cases, counterbalanced by a significant 
worsening in other cases (e.g. BAKER).

\section{Details of the best methods}

The GOAL method uses the similarity between the given refinement model and a list of templates generated by their fold recognition methods to select the best template and generate a new starting model, on which a refinement protocol similar to Princeton_Tigress MD-only is used. ${ }^{43}$ A number of minimization steps with gradually reduced positional restraints and short restrained MD simulations are performed. At difference with Princeton_Tigress, GOAL uses the AMBER ff14SB ${ }^{18}$ force field and explicit TIP3P water. ${ }^{44}$ GOAL's variant, LEE, uses modeling server models for initial clustering and the same refinement protocol. SVMQA and GOAL_COMPLEX use the same initial structures as GOAL, but perform a series of shorter MD simulations (5ns in total) using positional restraints. SVMQA complements the force-field with the DFIRE statistical energy term. ${ }^{29}$

The Seok method first predicts the inaccurate regions of the starting model, then it performs an extended structural sampling on those regions by normal mode analysis and secondary structure perturbations /hybridization, followed by short MD relaxation with a hybrid

potential. ${ }^{45}$ The difference between the Seok and Seok-server methods is that the latter submitted server predictions with no human intervention, while in the Seok predictions some of the final conformations are manually-selected and the error estimation results are modified after human inspection. ${ }^{45}$

The BAKER group performs a large scale conformational sampling using the Rosetta hybridization protocol followed by restrained MD simulations with the AMBER ff12SB force field and explicit TIP3P water on selected candidates. Depending on the distance of the 
starting structure from the target, either a high-resolution or a low-resolution protocol is used. In the former, Rosetta hybridization is only applied to rebuild local regions estimated to be less accurate, while in the low-resolution protocol, the iterative version is applied to rebuild the whole structure. For each of five selected Rosetta-refined models, 5 independent 10 ns-long MD simulations are performed and MD-refined models are ranked by the ensemble-average Rosetta energy.

The FEIG group uses by far the most extensive weakly restrained MD simulations (2-8 $\mu$ s per target) with explicit solvent and the recently re-parameterized CHARMM36m force field. ${ }^{21}$ From the MD structural ensembles a subset of structures is selected and averaged before finishing with detailed refinement of the local stereochemistry using “locPREFMD”. ${ }^{46}$

Kiharalab uses a combination of energy minimization, short and long MD simulations with the CHARMM22/CMAP force field and an implicit solvent.

As it can be seen from Figure 9, the GOAL method works best on targets with intermediate starting GDT_HA $(40<$ GDT_HA $<60)$ and those longer than 300 residues (see Figure 10 for an example). It also provides some well-refined targets in the GDT_HA $<40$ range where the initial structure is far from the experimental structure. Seok's approach ranks second in three categories (Starting GDT_HA $>60 ; 40<$ Starting GDT_HA $<60$ and $150<$ Residues $<300$ ) and on average has the best GDT_TS (Figure S5). BAKER remarkably comes on top in targets with extreme starting GDT_HA (either $>60$ or $<40$ ), submitting a number of strikingly refined structures (see Figure 11 for an example). FEIG's approach has on average the best GDT_HA scores (Figure 5) and ranks clearly first for smaller targets $(<150$ 
residues). This might reflect the fact that a strategy based purely on long MD simulations with accurate physics-based force fields and weak restrains (FEIG) is effective in exploring the conformational landscape of small proteins. However, large targets or those with low initial GDT_HA clearly benefit from combined methods where backbone-rebuilding methods are used before MD. Alternatively enhanced sampling MD has clear potential, as shown by the remarkable refinement of some more complex targets by the group Laufer_seed (Figure 10).

\section{Accuracy of model 1 compared to the other models}

CASP12 participants were allowed to submit 5 models per target. The additional submissions allow predictors to test different models, different versions of their methods or even completely different strategies. As in previous CASP experiments, we assessed the ability of different groups to identify their best models and protocols (tentatively model 1) out of the submitted ones. This is often an important task, as many approaches provide a number of structures and some methods include a final MD simulation, which generates a large ensemble of structures. A few methods based on structure selection and averaging have been developed to this end. ${ }^{11,42}$ GOAL, the top scoring approach according to the CASP12 score, uses a combination of trajectory averaging of atomistic explicit solvent MD simulations and energy minimization with an implicit solvent to address this issue. BAKER combines large scale conformational search using Rosetta with multiple 10ns-long MD simulations, run with the AMBER ff12SB force field and explicit TIP3P water. Structural averaging was used to obtain the representative conformation from the MD simulations.

In Figure 12, we report the ability of each group to correctly identify their best model (or protocol). On average, the groups were not able to correctly identify the best model (they did 
little better than random by identifying the best model in $30 \%$ of the cases). The best groups in this component of the analysis, MESHI and Kiharalab, succeeded in more than $60 \%$ of the targets. GOAL and Seok identified their best models/protocols in about $30 \%$ of cases, while BAKER identified the best model only in $24 \%$ of cases. The BAKER group would have ranked 2nd according to the CASP12 score if the best models (instead of Model 1) were considered, showing further potential for improvement of their protocol.

FEIG submitted as model 1 the predictions from the most comprehensive and expensive MDbased protocol. This reassuringly resulted in $36 \%$ of models 1 being the best model, showing that longer MD sampling indeed helps the refinement (and perhaps combining it with enhanced sampling algorithms might help even more).

\section{Progress since CASP11}

An important question addressed at every CASP meeting is the progress since last CASP. Comparison of the CASP12 results with those from two previous experiments is reported in Figure 13. The comparison shows the considerable improvement observed in CASP11 over CASP10, which, in turn, had already shown the most remarkable results since the inception of the refinement category in CASP8. CASP12 appears to consolidate the results of CASP11, showing similar global refinement when all submissions are considered. However, it is important to bear in mind that the targets are different in each CASP experiment, and we believe that the targets we released in this edition were more challenging, being both larger (including targets longer than 400 amino acids), more diverse and requiring more local refinement. In particular, six CASP12 targets were especially challenging: TR869, TR870, and TR898 had starting GDT-HA less than 30 and protein size larger than 100 aminoacids, 
while targets TR890, TR901 and TR905 had a starting GDT_HA less than 35 and protein size larger than 180 amino acids. It is thus reassuring to observe that the CASP12 predictions were as good as or better than CASP11 predictions.

\section{DISCUSSION AND CONCLUSIONS}

In the CASP12 refinement experiment, we have assessed the performance of 39 groups in refining 42 targets using a combination of standard Z-scores provided by the Structure Prediction Center.

We used a machine learning algorithm to select the weights for combining Z-scores so that they correlate in the best way with the manually curated rankings performed by 4 independent evaluators on $33 \%$ of the targets. Although the final ranking is similar to that obtained with the CASP11 score, we believe that the new score is better able to distinguish useful refinements (see SI Figures S11-S14).

CASP12 witnessed yet another step forward in the development of effective refinement algorithms. The methods were tested on the most diverse set of targets ever used in the refinement-CASP. Despite the challenging refinement target set, the CASP12 results proved to be on par with those from the previous CASP.

The best CASP12 methods can be roughly categorized as "conservative" (Seok and FEIG) and "adventurous" (BAKER and, to some extent, GOAL and similar methods from Lee's research group), with the first group of methods consistently refining the initial structures by a small amount and worsening few structures and the second group submitting some spectacular refinement but worsening a larger number of targets.

All best performing groups use restrained MD simulations, alone (FEIG and Kiharalab) or 
in combination with other approaches (GOAL, Seok and BAKER). Seven out ten run MD simulations with physics-based force fields (AMBER ff12SB, ff14SB and CHARMM36m) and explicit solvent, while three (Seok, Seok-server and Kiharalab) use a hybrid potential or an implicit solvent.

The GOAL method was the top performer according to the CASP12 score. This excellent result was obtained by combining an effective strategy to select the best templates to remodel the initial structures provided, followed by short MD runs with a good protein force field (AMBER ff14SB) and explicit solvent. As almost 30\% of the starting structures for refinement came from the GOAL server, there was a risk of diminishing returns for the starting model rebuilding strategy. Indeed, Figure 14 shows the difference in performance depending on the origin of the starting structure as measured by increase in GDT_HA. As expected, the median is clearly affected (and goes below zero) when the starting structure comes from the group itself. Still, even in these cases, GOAL was capable of some significant refinements, up to 13 GDT_HA units, showing the effectiveness of the MD refinement approach.

The BAKER method proved to be capable of generating striking refinements for difficult targets. The method ranked first on the targets in the difficult GDT_HA $<40$ category and in the GDT_HA $>60$ category. According to the authors, the method was in part limited by insufficient sampling and by failure of correcting sequence alignment errors ${ }^{47}$, showing a clear potential to emerge as a very robust and reliable refinement method across all categories. Seok's approach showed the ability to reliably refine almost all targets, albeit by a smaller amount. It is currently the best choice in terms of reliability. FEIG's approach does extremely well for small targets ( $<150$ residues) and provides the best GDT_HA improvement on average. However, being based on weakly 
restrained MD with a physics-based potential, it is penalized to a greater extent for missing structure-defining contacts (ligands, multimeric protein-protein contacts) and needs more extensive sampling and perhaps a different restraint strategy on larger and more difficult targets. Indeed, the use of positional restraints and plain (non-enhanced) MD keeps the refined structure close to the initial one and makes it particularly difficult to refine targets with low starting GDTHA. Interestingly, the authors convincingly show that increasing the MD sampling yields better refined models. ${ }^{46}$ An additional group that did better than the naïve method is Kiharalab, again with an MD-based protocol and an implicit solvent model.

It is worth bearing in mind that missing contacts are almost unavoidable in larger CASP targets, thus penalizing the strategies based on pure-MD. However, a special "high resolution" category might be introduced in future CASPs to address this problem.

Even among the second-tier groups, a few approaches delivered remarkably refined models for a subset of the targets, while performing modestly overall (see Figure 4 and 5). These include groups PKUSZ, Seminoles, Schroderlab and Laufer_seed. The latter uses MD with an enhanced sampling approach (MELD). ${ }^{26}$ Indeed, when considering the number of times their models ranked first for a target (SI Figure S7), or had a Z-score higher than 1.5 units (SI Figure S8), these groups perform as well as the top ones and provide a number of remarkably refined structures, see for instance Figure 10. With further development, any of these approaches has the potential of ranking very highly in future CASP refinement experiments, especially if a "high-resolution" category is introduced.

Although the combination of restrained MD simulations with accurate force-fields is clearly useful in protein structure refinement and has been adopted by most top-ranking groups, the larger and more difficult targets introduced in the CASP12 refinement challenge made clear the 
limits of this approach. When the starting structure is large or far from the target, restrained-MD works best when combined with methods that perform a more extensive sampling of the backbone conformational space. Indeed, limited sampling has been cited by the top performing research groups as a common cause of concern. According to their authors, both Feig's "pureMD" and Baker's hybrid method might have benefited from more extensive conformational sampling. In this respect, the use of enhanced sampling methods might be very beneficial, as shown by some remarkable refinements already obtained by some groups (such as Laufer_Seed). Methods combining coevolutionary data and MD simulations with hybrid force fields and enhanced sampling algorithms ${ }^{48}$ might also lead to further progress.

\section{Ideas for future CASP refinement experiments}

The experience in assessing the CASP12 refinement category has led us to propose a subdivision in two categories along the lines of high and low resolution refinement. The former category should include smaller targets providing all structure defining contacts (including ligands and co-factors). In this category, the use of starting structures different from the ones provided by the organizers should be strongly discouraged. This should provide a level ground and allow a fair comparison of MD-based and similar "pure refinement" approaches, including those avoiding explicit restraints and using enhanced-sampling algorithms, with hybrid methods. The low-resolution category (low starting GDT_HA) should cater to more adventurous methods and reward pipelines that include a systematic effort to locally rebuild the backbone of FM predictions with knowledge-based approaches that might do well even in the absence of important structure-defining contacts. An additional consideration arises by the lack of correlation of the MolProbity score with all the other quality scores. It is desirable that the role of this score in the future assessments is discussed before the start of the next experiment. 


\section{ACKNOWLEDGMENTS}

This paper is dedicated to Anna Tramontano.

The authors thank the CASP organizers and the Structure Prediction Center for their support. They thank and applaud the predictors for their participation, achievements and useful comments at the CASP12 meeting. We thank Lucas Siemons for useful discussions. Support for this work was provided by BBSRC ( $\mathrm{PhD}$ CASE Studentship to VO and London Interdisciplinary Doctoral Programme Fellowship to HY), A*STAR (Joint UCL-MAPS PhD studentship to $\mathrm{LH}$ ) and the Wellcome Trust (PhD studentship to LS). GS and FLG acknowledge EPSRC [grant no EP/M013898/1 ; EP/P011306/1 and EP/P022138/1] for financial support. VO and FLG acknowledge BBSRC [grant no BB/L502388/1] for financial support. HY acknowledges BBSRC [grant no. BB/M009513/1] for financial support.

\section{REFERENCES}

1 MacCallum, J. L., Hua, L., Schnieders, M. J., Pande, V. S., Jacobson, M. P., and Dill, K. A. Assessment of the protein- structure refinement category in CASP8. Proteins 2009; 77:66-80.

2 Zhang, Y. Protein structure prediction: when is it useful? Curr. Opin. Struct. Biol. 2009; 19:145-155.

3 Sliwoski, G., Kothiwale, S., Meiler, J., and Lowe, E. W. Computational Methods in Drug Discovery. Pharmacol. Rev. 2013; 66:334-395.

4 Becker, O. M., Dhanoa, D. S., Marantz, Y., Chen, D., Shacham, S., Cheruku, S., Heifetz, A., Mohanty, P., Fichman, M., Sharadendu, A., Nudelman, R., Kauffman, M., and Noiman, S. An integrated in silico 3D model-driven discovery of a novel, potent, and selective amidosulfonamide 5-HT1A agonist (PRX-00023) for the treatment of anxiety and depression. J. Med. Chem. 2006; 49:3116-3135.

5 Giorgetti, A., Raimondo, D., Miele, A. E., and Tramontano, A. Evaluating the usefulness of protein structure models for molecular replacement. Bioinformatics 2005; 21:ii72-6. 
6 Qian, B., Raman, S., Das, R., Bradley, P., McCoy, A. J., Read, R. J., and Baker, D. High-resolution structure prediction and the crystallographic phase problem. Nature 2007;450:259-64.

7 Nugent, T., Cozzetto, D., and Jones, D. T. Evaluation of predictions in the CASP10 model refinement category. Proteins 2014;82:98-111.

$8 \mathrm{Xu}, \mathrm{D} ., \mathrm{Zhang}$, J., Roy, A., and Zhang, Y. Automated protein structure modeling in CASP9 by I-TASSER pipeline combined with QUARK-based ab initio folding and FG-MD-based structure refinement. Proteins 2011;79:147-160.

9 Park, H., Ko, J., Joo, K., Lee, J., Seok, C., and Lee, J. Refinement of protein termini in template-based modeling using conformational space annealing. Proteins 2011; 79:2725-2734.

10 Raval, A., Piana, S., Eastwood, M. P., Dror, R. O., and Shaw, D. E. Refinement of protein structure homology models via long, all-atom molecular dynamics simulations. Proteins 2012; 80:2071-2079.

11 Mirjalili, V. and Feig, M. Protein structure refinement through structure selection and averaging from molecular dynamics ensembles. J. Chem. Theory Comput. 2013; 9:1294-1303.

12 Park, H., DiMaio, F., and Baker, D. CASP11 refinement experiments with ROSETTA. Proteins 2016; 84:314-322.

13 Feig, M. and Mirjalili, V. Protein structure refinement via molecular-dynamics simulations: What works and what does not? Proteins 2016; 84:282-292.

14 Lee, G. R., Heo, L., and Seok, C. Effective protein model structure refinement by loop modeling and overall relaxation. Proteins 2016; 84:293-301.

15 Della Corte, D., Wildberg, A., and Schröder, G. F. Protein structure refinement with adaptively restrained homologous replicas. Proteins 2016; 84:302-313.

16 Lindorff-larsen, K., Piana, S., Palmo, K., Maragakis, P., Klepeis, J. L., Dror, R. O., and Shaw, D. E. Improved side-chain torsion potentials for the Amber ff99SB protein force field. Proteins 2010; 78:1950-8.

17 Best, R. B., Buchete, N. V., and Hummer, G. Are current molecular dynamics force fields too helical? Biophys J 2008; 95:L07-L09.

18 Maier, J. A., Martinez, C., Kasavajhala, K., Wickstrom, L., Hauser, K. E., and Simmerling, C. ff14SB: Improving the Accuracy of Protein Side Chain and Backbone Parameters from ff99SB. J. Chem. Theory Comput. 2015; 11:3696-3713.

19 Robertson, M. J., Tirado-Rives, J., and Jorgensen, W. L. Improved Peptide and Protein Torsional Energetics with the OPLSAA Force Field. J. Chem. Theory Comput. 2015; 11:3499-3509.

20 Best, R. B., Zhu, X., Shim, J., Lopes, P. E. M., Mittal, J., Feig, M., and MacKerell, Jr., A. D. Optimization of the Additive CHARMM All-Atom Protein Force Field Targeting Improved Sampling of the Backbone $\varphi, \psi$ and Side-Chain $\chi$ land $\chi$ 2Dihedral Angles. J. Chem. Theory Comput. 2012; 8:3257-3273. 
21 Huang, J., Rauscher, S., Nawrocki, G., Ran, T., Feig, M., de Groot, B. L., Grubmüller, H., and MacKerell, A. D. CHARMM36m: an improved force field for folded and intrinsically disordered proteins. Nat Chem Biol 2017; 14:71-73.

22 Lindorff-Larsen, K., Piana, S., Dror, R. O., and Shaw, D. E. How Fast-Folding Proteins Fold. Science 2011; 334:517- 520.

23 Sutto, L., Marsili, S., and Gervasio, F. L. New advances in metadynamics. WIREs Comput Mol Sci 2012; 2:771-779.

24 Cavalli, A., Spitaleri, A., Saladino, G., and Gervasio, F. L. Investigating DrugTarget Association and Dissociation Mechanisms Using Metadynamics-Based Algorithms. Acc. Chem. Res. 2014; 8:277-285.

25 Pan, A. C., Weinreich, T. M., Piana, S., and Shaw, D. E. Demonstrating an Orderof-Magnitude Sampling Enhancement in Molecular Dynamics Simulations of Complex Protein Systems. J. Chem. Theory Comput. 2016; 12:1360-1367.

26 Perez, A., Morrone, J. A., Simmerling, C., and Dill, K. A. Advances in free-energybased simulations of protein folding and ligand binding. Curr. Opin. Struct. Biol. 2016; 36:25-31.

27 Samudrala, R. and Moult, J. An all-atom distance-dependent conditional probability discriminatory function for protein structure prediction. J. Mol. Biol. 1998; 275:895-916.

$28 \mathrm{Lu}, \mathrm{H}$. and Skolnick, J. A distance-dependent atomic knowledge-based potential for improved protein structure selection. Proteins 2001; 44:223-232.

29 Zhou, H. and Zhou, Y. Distance-scaled, finite ideal-gas reference state improves structure-derived potentials of mean force for structure selection and stability prediction. Protein Sci. 2002; 11:2714-26.

30 Chopra, G., Kalisman, N., and Levitt, M. Consistent refinement of submitted models at CASP using a knowledge-based potential. Proteins 2010; 78:2668-2678.

31 MacCallum, J. L., Perez, A., Schnieders, M. J., Hua, L., Jacobson, M. P., and Dill, K. A. Assessment of protein structure refinement in CASP9. Proteins 2011; 79:74-90. 32 Modi, V. and Dunbrack, R. L. Assessment of refinement of template-based models in CASP11. Proteins 2016; 84:260-281.

33 Zemla, A. LGA: A method for finding 3D similarities in protein structures. Nucleic Acids Res. 2003; 31:3370-3374.

34 Kryshtafovych, A., Monastyrskyy, B., Fidelis, K. CASP11 statistics and the prediction center evaluation system. Proteins. 2016; 84:15-19

35 Zemla, A., Venclovas, Č., Moult, J., and Fidelis, K. Processing and analysis of CASP3 protein structure predictions. Proteins 1999; 37:22-29.

36 Kryshtafovych, A., Monastyrskyy, B., and Fidelis, K. CASP prediction center infrastructure and evaluation measures in CASP10 and CASP ROLL. Proteins 2014; 82:7-13.

37 Mariani, V., Kiefer, F., Schmidt, T., Haas, J., and Schwede, T. Assessment of 
template based protein structure predictions in CASP9. Proteins 2011;79:37-58. 38 Chen, V. B., Arendall, W. B., Headd, J. J., Keedy, D. A., Immormino, R. M., Kapral, G. J., Murray, L. W., Richardson, J. S., and Richardson, D. C. MolProbity: allatom structure validation for macromolecular crystallography. Acta Crystallogr.

D. Biol. Crystallogr. 2010; 66:12-21

39 Olechnovič, K., Kulberkyt, E., and Venclovas, C. CAD-score: a new contact area difference-based function for evaluation of protein structural models. Proteins 2013;81:149-62.

40 Cong, Q., Kinch, L. N., Pei, J., Shi, S., Grishin, V. N., Li, W., and Grishin, N. V. An automatic method for CASP9 free modeling structure prediction assessment. Bioinformatics 2011; 27:3371-3378.

41 Jauch, R., Yeo, H. C., Kolatkar, P. R., and Clarke, N. D. Assessment of CASP7 structure predictions for template free targets. Proteins 69 Suppl 2007; 8:57-67.

42 Mirjalili, V., Noyes, K., and Feig, M. Physics-based protein structure refinement through multiple molecular dynamics trajectories and structure averaging. Proteins 2014; 82:196-207.

43. Khoury, G. A., Tamamis, P., Pinnaduwage, N., Smadbeck, J., Kieslich, C. A., Floudas, C. A. Princeton_TIGRESS: Protein geometry refinement using simulations and support vector machines. Proteins 2014; 82: 794-814.

44. Cheng, Q., Joung, I. and Lee, J. A Simple and Efficient Protein Structure Refinement Method J. Chem. Theory Comput 2017 in press DOI: 10.1021/acs.jctc.7b00470

45. Lee, G. R, Heo, L. and Seok, C. Simultaneous refinement of inaccurate local regions and overall structure in the CASP12 protein model refinement experiment Proteins (CASP special issue in press) 2017

46. Heo, L. and Feig, M. What makes it difficult to refine protein models further via molecular dynamics simulations? (CASP special issue in press) 2017

47 Ovchinnikov, S., Park, H., Kim, D., DiMaio, F. and Baker, D. Protein structure prediction using Rosetta in CASP12 (CASP special issue in press) 2017

48 Sutto, L., Marsili, S., Valencia, A. and Gervasio, F. L. From residue coevolution to protein conformational ensembles and functional dynamics. Proc Natl Acad Sci USA 2015; 112: 13567-13572.

\section{FIGURE CAPTIONS}

Figure 1. Target structures with inter-domain or inter-chain contacts. The domains to be refined are in cyan, additional domains are in gray. The interacting regions that were removed from the assessment are shown in red. These are: Q65F75 in TR868, I8-S21 in TR870, F107-A124 in TR876, V142-Y152 in TR866. In the case of TR887, the green region represents the swap segment added to the target structure from the second monomer.

Figure 2. Correlation between eight evaluation metrics for all targets and all 
submissions. Pair-wise scatter plots are in the left lower triangular part of the table; the correlation coefficients are in the upper one.

Figure 3. Normalized probability distributions of $\Delta$ GDT_HA differences between the refined and starting models for different target lengths (top row of graphs) and different starting GDT_HA (bottom row). Data for first submitted models are presented. Y-axis shows values of the probability density function (PDF) of the distribution.

Figure 4. Performance of CASP12 groups as evaluated by the differences in GDT HA scores between the refined and starting models. The data are shown for all targets (top panel) and for three target subclasses with different GDT_HA scores of starting models (i.e. different difficulties of original targets for tertiary structure prediction). Only models ranked as \#1 by the predictors are considered. The quartiles are shown as dotted lines in the violin plots. Groups are sorted according to decreasing delta_GDT_HA mean on all targets (top panel).

Figure 5. Overall performance by group as measured by RMSD, GDT_HA, SphGr, QCS and MolPrb Z-scores. Each panel shows boxplots of per-target Zscores for a specific measure. Groups are ordered left to right by the sum of RMSD Z-scores (top panel, higher is better). Missing predictions are assigned a value of -2 for each target. The number of submitted targets for each group are reported in grey on top of the box plots for MolPrb.

Figure 6. Cumulative group ranking for the eight selected metrics. The plot shows the number of times a group appears with a particular ranking in the best 10 models according to the various metrics considered separately. When a group is not in the best 10, we report whether the score is better or worse than that of the "nä̃e" submission. Thus, the sum of all bar heights for each group is always equal to eight (total number of metrics). Only groups appearing among the best 10 according to at least 2 metrics are shown.

Figure 7. Discrepancies between the GDT_HA and SphereGrinder scores for two different models on two refinement targets - TR882 and TR948. The target structure is colored blue, the starting model - gray and the prediction - based on per-residue distances between the corresponding $\mathrm{Ca}$ atoms in the superposition, ranging from green (improved over starting model) to yellow (no improvement) and red (worse). For clarity, part of the structure has been removed from target TR948. 
Figure 8. Overall performance by group as measured by the $\hat{S}_{\mathrm{CASP} 12}$ assessors score. Groups are ordered left-to-right by their rank (i.e., decreasing sum of $\hat{S}$ over all targets).

Figure 9. Overall performance by group as measured by the $\hat{S}_{\text {CASP12 }}$ assessors score on the targets grouped into three bins based on the starting model's GDT_HA (top row) and target size (lower row). Groups in each panel are ordered left-toright by their rank (decreasing sum of $\hat{S}_{\mathrm{CASP} 12}$ over all targets). Only the first submitted models are considered.

Figure 10. Some examples of notable refinement. The target structure is shown in blue, the starting model in gray and the prediction with a color scale based on per-residue distances between the corresponding $\mathrm{Ca}$ atoms in the superposition, ranging from green (improved over starting model) to yellow (no improvement) and red (worse).

Figure 11. Four predictions that improved over the starting model for target TR594 by more than 10 GDT_HA points The target structure is shown in blue, the starting model in gray and the prediction with a color scale based on $\triangle \mathrm{RMSD}$ ranging from green (improved over starting model) to yellow (no improvement) and red (worse).

Figure 12. Best model or method selection. The plot reports the percentage of submitted models \#1 that correspond to the best of the 5 submitted models. The numbers on top of the bars report the number of model $1 \mathrm{~s}$ corresponding to the best models (not all groups submitted models for all targets). The asterisks mark the CASP12 top performers.

Figure 13. Normalized probability distributions of delta-GDT HA and deltaRMSD scores in the latest three CASPs. Y-axis shows values of the probability density function (PDF) of the distribution.

Figure 14. Comparison of the refinement achieved by group 220 (GOAL) on targets for which the starting structure was provided by GOAL itself ("start") or by other groups ("not-start"). 